\title{
Gedankliche Höhenflüge über der Limmatstadt
}

\author{
Bruno Kesseli \\ Dr. med. et lic. phil., Chefredaktor
}

Die diesjährigen Zürcher Gesundheitstage auf dem Uetliberg standen unter dem vielversprechenden Titel "Märchen, Mythen und Bazare - und die Realität im Gesundheitsmarkt». Auch in der 11. Auflage dieser von der Ärztegesellschaft des Kantons Zürich organisierten Veranstaltung wurde den Teilnehmenden eine Reihe spannender Referate geboten. Daneben blieb reichlich Zeit für Diskussionen und persönliche Begegnungen.

Den fliegenden Teppich bemühte er für die Anreise auf den Uetliberg nicht, trotz des an 1001 Nacht erinnernden Tagungsmottos. Josef Widler, Präsident der organisierenden AGZ, macht einen sehr geerdeten Eindruck. Der Hausarzt, der auch im Zürcher Kantonsrat politisiert, ist bemüht, seine Politikerkolleginnen und -kollegen - bis hinauf zum Bundesrat - auf den Boden zu holen. Dies zeigt sich beispielsweise beim Thema Taxpunktwert. Josef Widler und sein Team setzen sich seit längerer Zeit hartnäckig, aber auch mit Fantasie und Humor, für einen Taxpunktwert ein, der eine angemessene Abgeltung der ärztlichen Leistungen gewährleistet. Noch lässt der Erfolg auf sich warten, aber steter Tropfen höhlt bekanntlich den Stein.

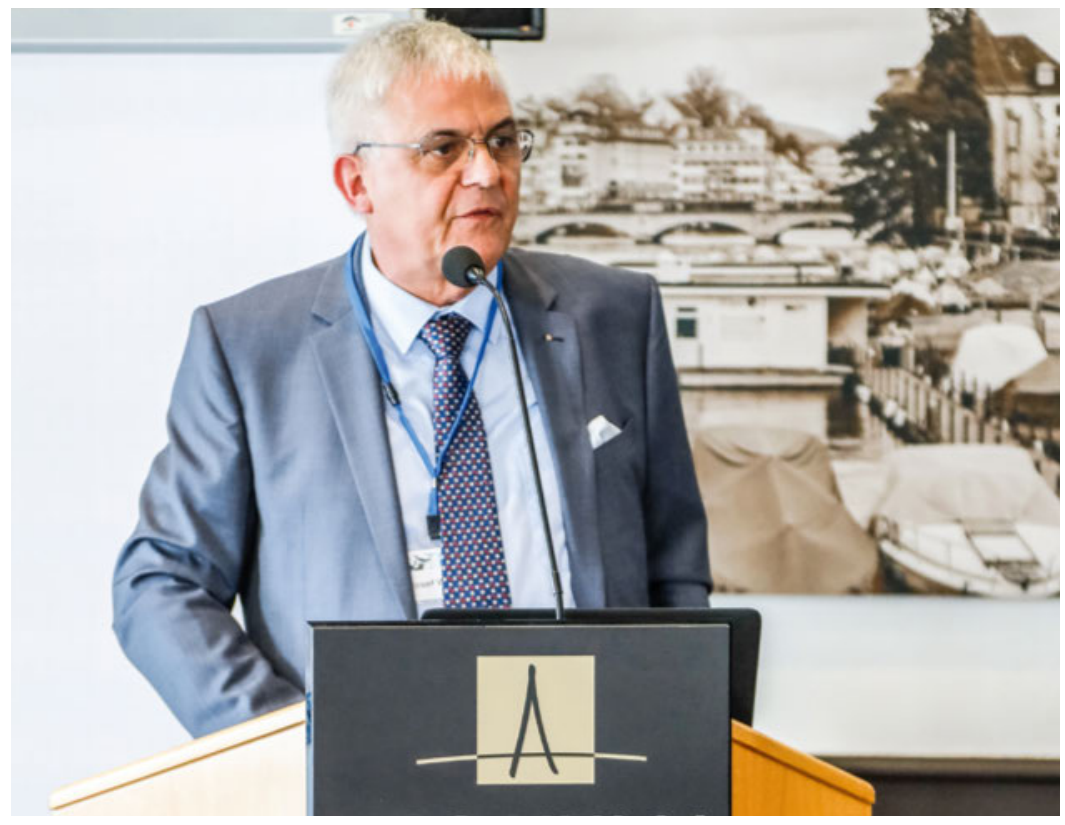

«Ein Arzt bleibt ein Arzt»: AGZ-Präsident Josef Widler bei seiner Eröffnungsansprache.

\section{Märchen im Gesundheitswesen}

In seinem Auftaktreferat zerpflückte der AGZ-Präsident verschiedene Mythen und Märchen. Demontiert wurde unter dem Schlagwort «Mythos Kostenneutralität» beispielsweise die Vorstellung, der «TARMEDKuchen» könne immer gleich gross bleiben. Faktoren wie demographischen Entwicklung, medizinischer Fortschritt, veränderten Ansprüche an die Versorgung oder auch die Forderung «ambulant vor stationär» entlarvten diese Behauptung als - eben ein Märchen. Zu dieser Gattung zählen für ihn auch «das Märchen von der immer weiter funktionierenden ambulanten Versorgung» oder das «Märchen von der angebotsinduzierten Nachfrage». Richtige Leistungssteuerung erfolgt aus Widlers Sicht über Guidelines, Netzwerke, den sinnvollen Einsatz von e-Health und vor allem den Einbezug des Patienten. «Ein Arzt bleibt ein Arzt», bilanzierte er zum Schluss und sprach damit auch einen mit der medizinischen Profession verbundenen «Heileraspekt» an, der sich weder an Facebook noch an Dr. Google delegieren lasse.

\section{Mythos Eigenverantwortung}

Mit dem «Mythos Eigenverantwortung für die Gesundheit» setzte sich in seinem Vortrag Wolfgang Bürgstein auseinander. Der Generalsekretär der Schweizerischen Nationalkommission Justitia et Pax ist Theologe und Ökonom und hat seine Dissertation zu diesem Thema verfasst. Er näherte sich dem Begriff der Eigenverantwortung im Zusammenhang mit Gesundheitskosten, Medizin und Gesundheit sehr systematisch aus einer sozialethischen Perspektive. Dabei stellte er unter anderem fest, dass eine allgemein verbindliche Orientie- 
rung und ein gemeinsames Verständnis von Gesundheit und Wohlbefinden heutzutage weitgehend fehlten. Im aktuellen gesundheitspolitischen Kontext erfolgt der Ruf nach mehr Eigenverantwortung gerne im Sinne einer gefälligen rhetorischen Floskel. Bürgstein sprach in diesem Zusammenhang von einem "moralisch aufgeladenen Verantwortungs-Pathos» und stellte fest, dass die Eigenverantwortung im Konkreten oft zur Überforderung wird, da sie Freiheit, Wissen und Alternativen voraussetzt. Diese Voraussetzungen sind im Einzelfall aber in sehr unterschiedlichem Mass gegeben. In seinem Fazit unterstrich Bürgstein denn auch, dass Eigenverantwortung für die Gesundheit nicht mit Alleinverantwortung gleichgesetzt werden dürfe. Sie sei als Befähigung zur «Eigenmächtigkeit» zu verstehen und sinnvollerweise Bestandteil eines integrativen Verantwortungskonzepts. Ein solches Konzept gehe weit über gesundheitsrelevante Ratschläge hinaus und umfasse auch Faktoren wie Bildung, Arbeitswelt, Soziales, Umwelt und weitere.

\section{Alternativmedizin «voll von Märchen»}

Dass sich mit der Märchenmetaphorik im Bereich der Alternativ-, aber auch der Schulmedizin rhetorisch effektvoll arbeiten lässt, demonstrierten Edzard Ernst und Johann Steurer in ihren jeweiligen Referaten. Ernst, der an der Universität Exeter (GB) über viele Jahre einen Lehrstuhl für Komplementär- und Alternativmedizin innehatte, ist ein bekannt kritischer Geist. Mit seinen Arbeiten hat er sich in alternativmedizinischen Krei-

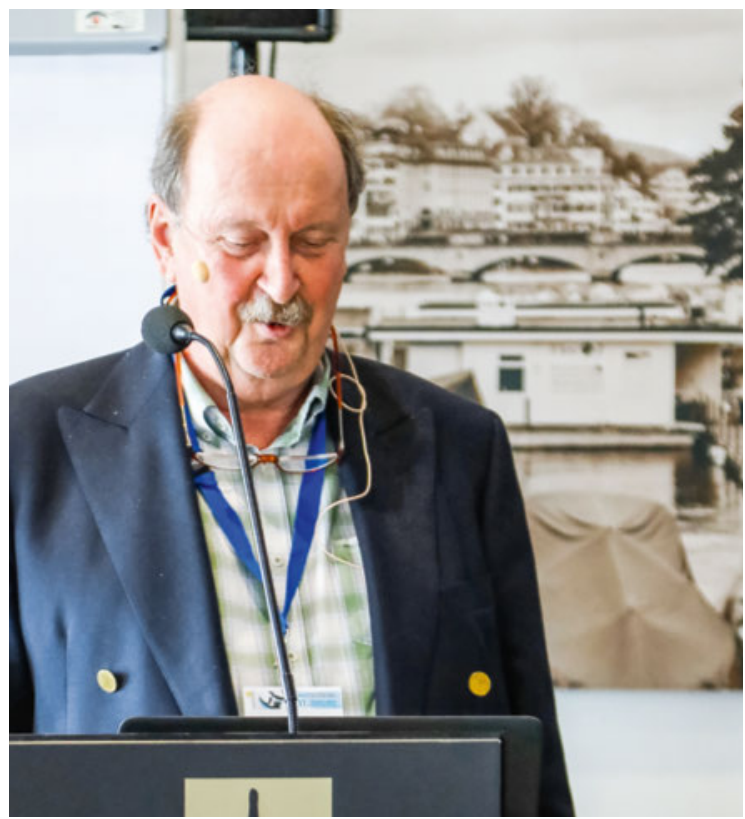

Kritischer Geist: Edzard Ernst, emeritierter Professor für Komplementär- und Alternativmedizin an der Universität Exeter. sen kaum Freunde gemacht. Sein pointierter Vortrag, der im Publikum mehrfach Heiterkeitsausbrüche provozierte, stellte - erwartungsgemäss - den meisten Methoden der Komplementär- und Alternativmedizin ein vernichtendes Zeugnis aus. Von den angeführten Beispielen erhielten lediglich die Phytotherapie und die Akupunktur punktuell eine zurückhaltend-positive Bewertung. Die Alternativmedizin, so seine Schlussbilanz, sei voll von Märchen. Keines der Verfahren sein nebenwirkungsfrei, und abgesehen von wenigen Fällen sei es sehr zweifelhaft, ob der Nutzen den Schaden überwiege.

\section{Lückenhafte Evidenz}

Johann Steurer, Direktor des Horten-Zentrums für praxisorientierte Forschung und Wissenstransfer der Universität Zürich, unterzog in seinem Referat die Evidenzbasis der Schulmedizin einer kritischen Prüfung Dass es zu vielen Fragen in der Medizin keine Studien und damit keine Evidenz gibt, ist bekannt. Doch selbst dort, wo es sie gibt, sind im Einzelfall die entscheidenden Fragen nicht immer beantwortet, wie Steurer an einer Reihe von Beispielen zeigte. So belegen die verfügbaren Daten, dass zwei Drittel der Patienten mit einem engen Spinalkanal von einer Operation profitieren, während ein Drittel keine Besserung erfährt. Zentral für den Operationsentscheid wäre es, zu wissen, welchen Patienten es nach dem Eingriff bessergeht. Doch genau diese Frage lässt sich nicht evidenzbasiert beantworten.

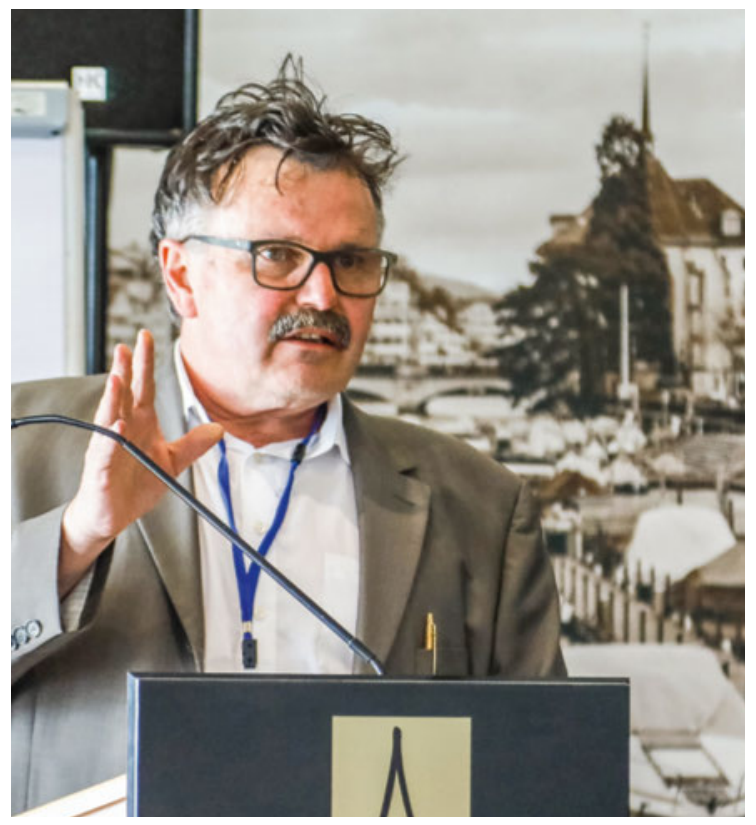

Johann Steurer, Direktor des Horten-Zentrums der Universität Zürich, sprach zur Evidenzbasis der Schulmedizin. 


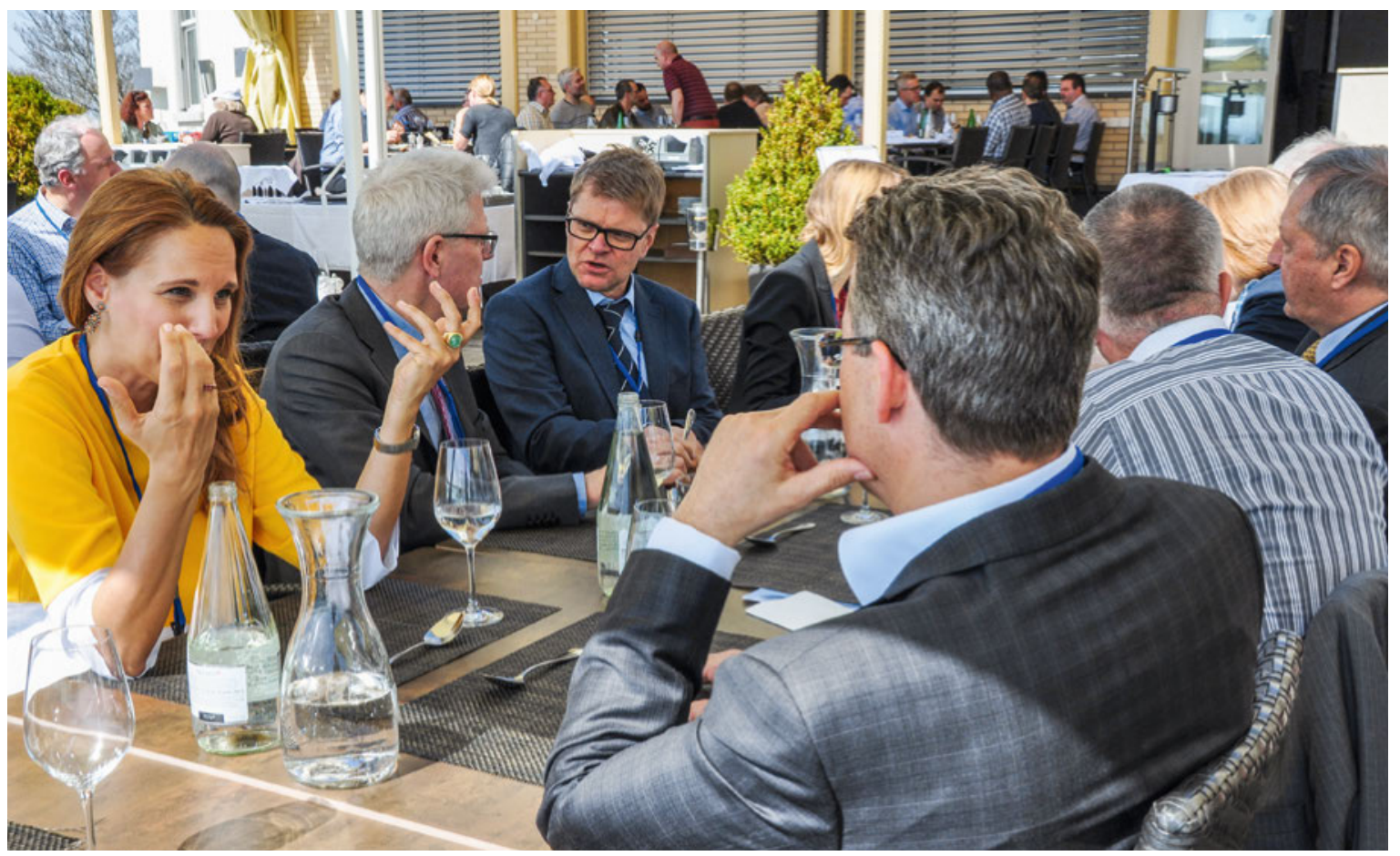

Die Zürcher Gesundheitstage bieten viel Raum für den Austausch unter den Teilnehmenden.

Selbst Metaanalysen zu bestimmten Fragen würden sich teilweise widersprechen, etwa zum Nutzen von Vitamin D in der Frakturprophylaxe oder zur Wirksamkeit von Antidepressiva. Die Zukunft, so Steurer, liege in der individuellen Risikoabschätzung, und Computer würden dabei eine entscheidende Rolle spielen. Verschiedene zentrale ärztliche Qualitäten werde der Computer aber nicht übernehmen können. Patienten wünschten sich Empathie, Zuwendung und Zeit. In diesem Bereich könne die Schulmedizin auch von der Komplementärmedizin lernen.

\section{Medikamente als Kostentreiber?}

Auch am zweiten Symposiumstag liessen die Redner das Tagungsmotto immer wieder explizit aufleben. Lorenz Borer, Head Market Access \& Compliance bei Novartis Schweiz, hatte unter anderem den Mythos im Fokus, dass Arzneimittel die wesentlichen Kostentreiber im Gesundheitswesen seien. Dieser Mythos ist gemäss Borer widerlegt. Der Anteil der Medikamente an den Schweizer Gesundheitsausgabe liege unter 10\% und der Kostenanstieg der Medikamente sei trotz hoher Innovation unterdurchschnittlich. Borer stellte interessante neue Finanzierungsmodelle vor, die auf Wirkung und Nutzen für den Patienten ausgerichtet sind. Auch auf diese Weise könnten Einsparungen erzielt werden, sofern die verschiedenen Stakeholder zur Zusammenarbeit bereit seien.

\section{Die Tricks der Gesundheitsbranche}

Geradezu ein Feuerwerk an Mythen präsentierte Werner Bartens der Zuhörerschaft auf dem Uetliberg. Der Arzt, Wissenschaftsjournalist und erfolgreiche Buchautor entzauberte sie in der Tradition eines kritischhinterfragenden Journalismus mit witziger Rhetorik auch er hatte die Lacher damit auf seiner Seite. Neben Trends wie «Entschlackung», die er als pseudowissenschaftlich bezeichnete, nahm er auch die Gesundheitsindustrie als Ganzes ins Visier. Für eine Wachstumsbranche sei es "doof», wenn die Leute nicht kränker würden. Deshalb seien Tricks beliebt wie die Schaffung neuer Krankheiten, das Senken von Grenzwerten oder die Propagierung nutzloser und überflüssiger Therapien. Für Ärzte sei es generell schwierig, nichts zu tun. Bewegungen wie "Choosing wisely», die aus der Medizin selbst kommen, könnten sie darin unterstützen, unnötige Interventionen zu vermeiden. Wie sich zum Schluss vielleicht etwas überraschend zeigte, liegt die Essenz des ärztlichen Wirkens auch für den meist mit einer gehörigen Prise Sarkasmus argumentierenden Bartens in Tugenden wie Sich-Zeit-Nehmen, Zuwendung und Barmherzigkeit.

\section{Intelligentere Regulierungen gefragt}

Der interessanten Frage, was denn hinter der Ablehnung staatlicher Interventionen (im Gesundheitswesen) stecke, ging Stefan Spycher in seinem Vortrag nach. 


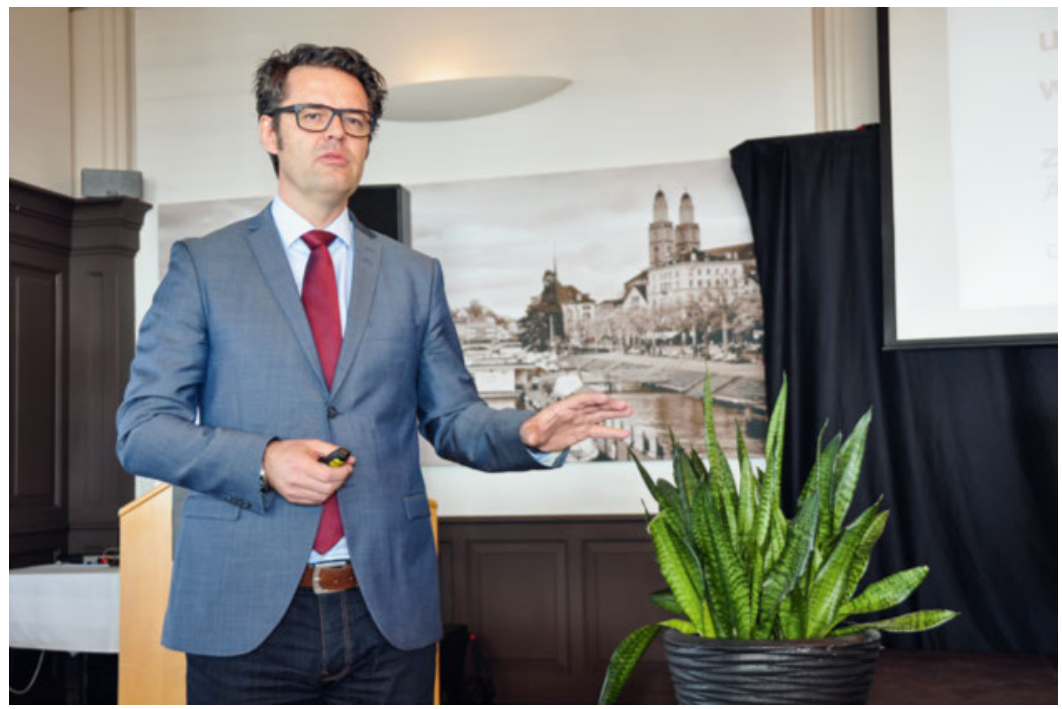

«Intelligentere Regulierungen»: Stefan Spycher zur Rolle des Staats im Gesundheitswesen.

bkesseli[at]emh.ch
Der Vizedirektor des Bundesamts für Gesundheit skizzierte zunächst verschiedene "Bilder vom Staat», die in den Köpfen der Bürger existieren und widmete sich auch dem Zusammenspiel von «Staat und Markt». Wieviel es vom einen und vom anderen für ein gut funktionierendes Staatswesen braucht, ist seit Adam Smith umstritten. Das Schweizer Gesundheitssystem mit seinem «regulierten Wettbewerb» erhält von der Bevölkerung und auch von internationalen Expertengremien grundsätzlich gute Noten. Angesichts der bekannten Herausforderungen wie demographischer Wandel, Zunahme chronischer Krankheiten oder Finanzierungsprobleme liegt der Ruf nach mehr Staat nahe. Spycher plädierte dagegen nicht für mehr staatliche Interventionen, sondern für intelligentere Regulierungen. Dabei sollte der Staat seiner Meinung nach nicht selbst produzieren und umsetzen, sondern die Spielregeln bestimmen und allenfalls finanzielle Unterstützung bieten.

\section{Bazar der Ideen}

Den Schlusspunkt der Veranstaltung setzte in guter Uetliberg-Tradition Ludwig Hasler. Einmal mehr verstand es der Philosoph und Publizist, zentrale Aussagen der Referenten aufzugreifen, in geistreicher und humorvoller Weise zu kommentieren und mit eigenen Gedanken zu verbinden. Damit schloss er eine Tagung ab, die in Anlehnung an ihr Motto als anregender «Bazar der Ideen» in Erinnerung bleiben wird. Es wäre dem Gesundheitsmarkt sicher zuträglich, wenn die eine oder andere dieser Ideen Wirkung entfalten könnte.

\section{Bildnachweise:}

Fotos 1-3 (Josef Widler / Edzard Ernst / Johann Steurer): AGZ / Delia Frauenfelder. Fotos 4 und 5 (Teilnehmende im Gespräch / Stefan Spycher): AGZ. 